Luc Keunings, Des polices si tranquilles. Une histoire de l'appareil policier belge au XIX ${ }^{e}$ siècle

Louvain, Presses Universitaires de Louvain, collection « Histoire, justice, sociétés », 2009, 299 pp. , ISBN 9780754656128.

Arnaud-Dominique Houte

\title{
OpenEdition
}

Journals

Édition électronique

URL : https://journals.openedition.org/chs/1263

DOI : $10.4000 /$ chs. 1263

ISSN : 1663-4837

Éditeur

Librairie Droz

Édition imprimée

Date de publication : 1 mai 2011

Pagination : 146-148

ISBN : 978-2-600-01515-8

ISSN : 1422-0857

Référence électronique

Arnaud-Dominique Houte, "Luc Keunings, Des polices si tranquilles. Une histoire de l'appareil policier belge au XIXe siècle », Crime, Histoire \& Sociétés / Crime, History \& Societies [En ligne], Vol. 15, n 1 | 2011, mis en ligne le 06 mars 2013, consulté le 23 mars 2022. URL : http://journals.openedition.org/chs/ 1263 ; DOI : https://doi.org/10.4000/chs.1263

Ce document a été généré automatiquement le 23 mars 2022

(C) Droz 


\section{Luc Keunings, Des polices si tranquilles. Une histoire de l'appareil policier belge au XIX siècle}

Louvain, Presses Universitaires de Louvain, collection « Histoire, justice, sociétés », 2009, 299 pp. , ISBN 9780754656128.

\section{Arnaud-Dominique Houte}

\section{RÉFÉRENCE}

Luc Keunings, Des polices si tranquilles. Une histoire de l'appareil policier belge au XIX ${ }^{e}$ siècle, Louvain, Presses Universitaires de Louvain, collection « Histoire, justice, sociétés ", 2009, 299 pp. , ISBN 9780754656128.

1 Depuis ses premiers travaux, au début des années 1980, Luc Keunings est bien connu du petit milieu des spécialistes de l'histoire policière. Ses nombreux articles et son mémoire de licence resté inédit n'étaient cependant accessibles qu'aux chercheurs les mieux informés. Ils auraient pu sombrer dans l'oubli si une nouvelle génération de jeunes historiens n'avait pas repris le dossier de l'histoire de la sécurité. Citons ainsi, parmi les héritiers proches ou lointains de Luc Keunings, Margo De Koster et Axel Tixhon, pour le $\mathrm{XIX}^{\mathrm{e}}$ siècle, mais aussi Jonas Campion et Benoît Majerus pour le XX ${ }^{\mathrm{e}}$ siècle.

2 Si ces jeunes spécialistes de l'histoire policière et gendarmique belge ont pu fréquenter et prolonger les travaux de Luc Keunings, leurs camarades français ou suisses, pour ne citer qu'eux, connaissent très mal - c'est un euphémisme - un système policier belge qui emprunte pourtant beaucoup aux modèles étrangers et qui leur semblera étrangement familier.

Il faut donc saluer la publication, dans l'excellente collection dirigée par Xavier Rousseaux, de cette synthèse éminemment pédagogique. Disons-le tout de suite: l'ouvrage séduira tous les historiens du XIX ${ }^{\mathrm{e}}$ siècle, quelle que soit leur spécialité. Par la 
clarté de son plan et par l'exceptionnelle richesse de ses annexes, il constitue à bien des égards un modèle de manuel universitaire.

4 Le titre choisi fait écho à une certaine image traditionnelle de la Belgique, "pays si tranquille» dans lequel la police tiendrait une place quasiment négligeable. Luc Keunings fait immédiatement un sort à cette image d'Épinal qui ne résiste pas à l'examen des mutations du XIX ${ }^{e}$ siècle. La Belgique, c'est le pays de la croissance urbaine et industrielle, de la contestation ouvrière et socialiste, des conflits religieux, des vagabonds et des mendiants, etc. Un pays où, c'est précisément la thèse maîtresse du livre, la police est appelée à jouer un rôle de plus en plus significatif.

Consacrée aux premières décennies du tout neuf État belge, la première partie offre une présentation générale d'un appareil policier très diversifié. Fille d'une révolution, la jeune monarchie fait le pari du libéralisme. Elle maintient un instrument de surveillance politique, la Sûreté, mais elle confie la gestion de la sécurité aux municipalités. Avec des résultats variés: si Bruxelles s'inspire du modèle londonien pour former une police moderne, de nombreuses villes restent dépourvues de véritables forces de sécurité. Sans parler des campagnes, où les gardes champêtres sont unanimement critiqués pour leur incompétence et pour leur partialité. Le choix libéral de la Belgique se manifeste encore plus clairement à travers la valorisation de la " garde civique ». Cette milice citoyenne rassemble les «bons pères de famille », selon l'expression consacrée. Garante de l'ordre et de la propriété, elle n'est cependant pas tout à fait fiable. Peu dynamique, inégalement organisée selon les lieux, elle manque de discipline.

6 Aussi le maintien de l'ordre et la sécurité reposent-ils de plus en plus sur les institutions militaires, à commencer par la gendarmerie. Petite-fille de la gendarmerie impériale napoléonienne et fille de la maréchaussée néerlandaise, cette arme d'élite a été épurée et remodelée sur la base d'une stricte culture militaire et d'un puissant esprit de corps. Réputée pour son efficacité, elle reste néanmoins trop peu nombreuse pour satisfaire des besoins croissants. À raison d'un gendarme pour 3329 habitants au milieu du siècle, le taux d'encadrement de la population est presque deux fois plus faible en Belgique qu'en France. La monarchie doit donc encore compter sur l'armée, qui garde un rôle central dans le maintien de l'ordre.

7 Cette première époque s'achève, selon Luc Keunings, au cours des années 1870. Les tensions entre catholiques et libéraux, l'émergence des socialistes et même des anarchistes, la montée en puissance du mouvement social, rendent beaucoup plus urgente la question du maintien de l'ordre, tandis que la crise économique, l'urbanisation et l'industrialisation font apparaître des "classes dangereuses » et un climat d'inquiétude qui renforce la demande de sécurité.

8 Les temps changent, les outils s'adaptent. Si aucune réforme d'envergure ne bouleverse un système policier très stable, la hiérarchie des polices se déplace. Préservée en tant que symbole politique, la garde civique est marginalisée dans les faits. Les gardes champêtres cèdent une bonne part de leurs prérogatives à une gendarmerie toujours plus puissante. Triplement des effectifs entre 1875 et 1914, quadruplement des dépenses budgétaires : l'arme devient hégémonique dans le domaine de la police rurale, mais elle tient également le haut du pavé dans les villes et dans le maintien de l'ordre.

9 Revers de la médaille, la gendarmerie cristallise sur son uniforme l'essentiel de la colère populaire. Plus que l'armée, qui reste également mobilisée lors des grands conflits sociaux, mais qui se tient plutôt en seconde ligne. Plus que la Sûreté, qui cède 
aux gendarmes «déguisés en bourgeois » le premier rôle dans les opérations de renseignement politique. Plus, enfin, que les polices urbaines, qui continuent à se développer, mais qui suivent désormais, pour la plupart d'entre elles, un modèle plus militaire que libéral.

Ébauche de centralisation, professionnalisation, militarisation... Voici quelques-unes des principales tendances qui traversent le XIX ${ }^{e}$ siècle des polices belges. Mais Luc Keunings insiste à juste titre sur une autre caractéristique majeure: le sousfinancement des questions de sécurité, qui restent un sujet secondaire dans la vie politique belge.

11 Aussi claire que dense, cette synthèse générale n'occupe qu'une petite centaine de pages. L'effort de concision est d'autant plus louable qu'il permet de publier, souvent dans leur intégralité, près de cent cinquante documents. Depuis la disparition déjà ancienne des vénérables collections «U2 » d'Armand Colin et «Archives Julliard », on avait rarement vu un tel foisonnement! Des textes officiels, bien sûr, mais aussi des rapports, des courriers, quelques extraits de mémoires, ainsi que de nombreux articles de presse, discours politiques, etc. Quand on y ajoute de riches illustrations, on comprend mieux l'importance et l'originalité d'un ouvrage qui livre sa matière brute et qui offre ainsi une formidable occasion de revenir, textes en main, sur les hypothèses et sur les conclusions développées dans la première partie.

Quelques réflexions viennent ainsi à l'esprit du lecteur, qui pourra s'interroger sur la spécificité des différentes polices urbaines et sur l'originalité, en particulier, du modèle bruxellois. Les rapports des commissaires et des officiers livrent également des indications fort suggestives sur les techniques du maintien de l'ordre et sur les formes du renseignement politique. On trouvera ici des documents tout à fait passionnants des mines d'or pour commentaires de documents.

13 Pour s'en tenir à un thème qui est rapidement esquissé, peut-être trop vite éludé, les annexes livrent une manne d'informations sur les représentations de la gendarmerie et sur sa culture professionnelle, telle qu'elle est révélée par un certain nombre de discours et de témoignages. Question centrale : si l'on comprend bien que les ouvriers s'indignent de la violence parfois meurtrière des gendarmes, il n'en faut pas moins remarquer qu'ils cohabitent les uns avec les autres. Confinés dans le "splendide isolement » de leurs casernes, les gendarmes sont-ils vraiment retranchés du monde extérieur? Dans quelle mesure leur pratique professionnelle s'infléchit-elle loin du regard des officiers? Quelle est l'importance du facteur linguistique dans cette histoire? On voit que le livre de Luc Keunings ouvre de nombreuses pistes de recherches.

14 Ajoutons, pour faire bonne mesure, une chronologie qui ne pèche pas par excès de détail, une liste des différents ministres concernés par la sécurité au XIX siècle et des principaux chefs de la police et de la gendarmerie. Même l'index des personnages cités dans le livre se prolonge par un précieux dictionnaire qui guidera en particulier les Français, généralement piètres connaisseurs de l'histoire politique belge. Répétons-le sans hésiter : cette passionnante " histoire de l'appareil policier belge » rendra bien des services à tous ceux qui s'intéressent à l'histoire du XIX siècle européen et à ceux qui croisent, de près ou de loin, les questions de sécurité. 


\section{AUTEURS}

ARNAUD-DOMINIQUE HOUTE

Université de Paris-Sorbonne

arnaudhoute@aol.com 